\title{
Smart cities e pandemia: tecnologias digitais na gestão pública de cidades brasileiras
}

\author{
Tharsila Maynardes Dallabona Fariniuk 12 \\ 1 Pontifícia Universidade Católica do Paraná, Curitiba / PR - Brasil \\ ${ }^{2}$ Centro Universitário Unifacear / Curso de Arquitetura e Urbanismo, Curitiba / PR - Brasil
}

\begin{abstract}
A pandemia da COVID-19 vem afetando o modo de vida nas cidades. Em alguns países - como o Brasil - a curva epidemiológica cresce significativamente, exigindo respostas rápidas da administração pública. Esta pesquisa, conduzida em abril de 2020, visa caracterizar a utilização de ferramentas digitais na adaptação das cidades brasileiras à pandemia, baseada no conceito de smart city. Para tanto, buscou-se traçar um panorama da adoção de ferramentas digitais pela administração pública nas cem maiores cidades brasileiras, como resposta aos desafios impostos pela situação pandêmica, contribuindo para efetivar o isolamento ou para adaptar o funcionamento de atividades. Os resultados indicam a aplicação de tais estratégias em $83 \%$ das cidades pesquisadas, segundo a distribuição geográfica de casos da doença confirmados. A concentração das iniciativas sugere que a adoção da tecnologia acompanha a previsão da curva de infecção, e aponta para a tendência de adoção das estratégias em locais de contexto historicamente favorável à inovação. Característica fundamental das cidades inteligentes, o recurso à tecnologia digital na otimização dos serviços indica que as cidades pesquisadas estão, em algum nível, seguindo uma tendência mundial.
\end{abstract}

Palavras-chave: COVID-19; smart cities; tecnologias digitais; gestão pública.

\section{Smart cities y pandemia: tecnologías digitales en la gestión pública de ciudades brasileñas}

La pandemia de COVID-19 ha estado afectando la forma de vida en las ciudades. En algunos países -como Brasilla curva epidemiológica crece significativamente, lo que requiere respuestas rápidas de la administración pública. Esta investigación, realizada en abril de 2020, tiene como objetivo caracterizar el uso de herramientas digitales en la adaptación de las ciudades brasileñas a la pandemia, a la luz del concepto de ciudad inteligente. Para ello, buscamos esbozar un panorama de la adopción de estas herramientas por parte de la administración pública en las cien ciudades brasileñas más grandes, como una forma de responder a los desafíos impuestos por la situación de pandemia, contribuyendo a efectuar el aislamiento o adaptar el desempeño de las actividades. Los resultados indicaron la presencia de tales estrategias en el $83 \%$ de las ciudades encuestadas. La concentración de las iniciativas sugiere que la adopción de la tecnología sigue el pronóstico de la curva de infección, y señala la tendencia a adoptar estrategias en lugares con un contexto históricamente favorable a la innovación. Característica fundamental de las ciudades inteligentes, el uso de tecnología digital en la optimización de los servicios indica que las ciudades encuestadas están siguiendo, en algún nivel, una tendencia mundial.

Palabras clave: COVID-19; smart cities; tecnologías digitales; gestión pública.

\section{Smart cities and the pandemic: digital technologies on the urban management of Brazilian cities}

COVID-19 has been affecting the way of life in cities. The pandemic curve grows significantly in some countries, such as Brazil, requiring rapid responses from the public administration. This research, conducted in April 2020, characterizes the use of digital tools in adapting Brazilian cities to the pandemic in light of the concept of smart cities, presenting a panorama of the current situation. The results indicated that $83 \%$ of the cities surveyed used digital tools in measures to fight the pandemic, such as increasing social distancing and adapting public services. The concentration of initiatives tends to follow the geographic distribution of confirmed cases, i.e., digital tools are more used in places where the pandemic curve is more accentuated. Also, cities that are historically more open to innovation demonstrated a heavier use of digital technologies and strategies to fight the pandemic. Finally, the results indicate that the largest Brazilian cities follow, at some level, the trends of digital optimization observed worldwide. Keywords: COVID-19; smart cities; digital technologies; public management. 


\section{INTRODUÇÃO}

O avanço das Tecnologias de Informação e Comunicação, cada vez menores em tamanho e mais sofisticadas, vem alterando as relações urbanas. Ao mesmo tempo, tais tecnologias são potencialmente capazes de ampliar o reconhecimento de problemáticas urbanas, muitas vezes invisíveis. Trata-se do que Amin e Thrift (2002, p. 8) denominam como "um novo urbanismo", em que a relação homemmáquina traduz a complexidade crescente dos aglomerados urbanos.

Mais recentemente, o termo smart city emergiu como definição relevante para esse processo. Embora não exista um consenso sobre o conceito (Angelidou, 2014; Albino, Berardi, \& Dangelico, 2015; AlDairi \& Tawalbeh, 2017; Bibri \& Krogstie, 2017; Cassandras, 2016), o termo é geralmente utilizado para definir cidades que utilizam ferramentas digitais com o propósito de automatizar sistemas e serviços (AlDairi \& Tawalbeh, 2017; Llacuna, Colomer-llinàs, \& Meléndez-Frigola, 2015), com ênfase na sustentabilidade, sensoriamento e colaboração (Abella et al., 2015) e finalidade de otimização de recursos e precisão da oferta de serviços públicos (Salgado, 2016). Outros fatores, como resiliência urbana, adaptabilidade e capacidade governamental, são também considerados no conceito. Essas características vieram novamente à tona recentemente, quando uma situação em especial colocou à prova a estruturação das cidades mundo afora: a pandemia da COVID-19.

Identificada inicialmente na China, em 2019, a pandemia vem afetando o modo de vida nas cidades de forma global. Até o momento de redação deste texto (abril de 2020), já são mais de 3,2 milhões de casos confirmados no mundo (TradingView, como citado em Organização Mundial da Saúde [OMS], 2020). Em alguns países já se observa o declínio do número de casos e óbitos; enquanto em outros, a curva cresce significativamente - caso do Brasil -, exigindo respostas rápidas da administração pública.

Sakellarides (2020) comenta que, à medida que se observa o declínio da curva pandêmica, é preciso aproveitar as vitórias como uma oportunidade de consolidar políticas públicas e ações administrativas. O autor afirma que é preciso avançar efetivando a inteligência comunitária, especialmente no que diz respeito a uma interconectividade aprimorada que auxilie no monitoramento e na interpretação de dados relativos à saúde. Além disso, a oportunidade do tratamento de dados, em situações como a atual, revela que há bastante o que refletir sobre o comportamento humano diante do rearranjo de mobilidades, presencialidade e conexões entre indivíduos (Oliver et al., 2020).

Esse é o contexto norteador desta pesquisa, do qual emergem alguns questionamentos: o conceito de smart city, compreendido, entre outros fatores, como o aprimoramento da gestão por meio de ferramentas digitais e fluxos informacionais, pode ser vislumbrado nas ações de adaptação à situação pandêmica? Quais práticas brasileiras atuais podem sugerir a manifestação do fenômeno de espaços urbanos e governanças mais inteligentes, traduzidas no entendimento dos gestores sobre o potencial tecnológico na articulação da resiliência urbana?

Desse modo, o objetivo desta pesquisa é caracterizar a utilização de ferramentas digitais na adaptação das cidades brasileiras à situação pandêmica da COVID-19, à luz do conceito de cidade inteligente. A discussão gira em torno de duas hipóteses antitéticas: a) a adoção da tecnologia acompanhou - ou até mesmo antecipou - a previsão de crescimento da curva de infecção, o que refletiria um aspecto fundamental das cidades inteligentes: a resposta da administração pública com a utilização das tecnologias como ferramentas de potencialização da resiliência; e b) a adoção da tecnologia não serviu para antecipar os acontecimentos, mas sim como resposta, a posteriori, ao aumento das curvas de infecção nos municípios. Nesse caso, a velocidade de resposta se explicaria pela exigência imediatista da situação enfrentada. 
Nesta investigação, traçou-se um panorama da adoção de ferramentas digitais por parte da administração pública como forma de responder a certos desafios impostos pela situação: o da efetivação do isolamento e o da adaptação de atividades. O panorama foi delineado por intermédio do levantamento de dados nas cem maiores cidades brasileiras.

Esta pesquisa origina-se de uma série de estudos anteriores a respeito da construção fenomenológica do conceito de smart cities no Brasil. Utiliza-se, aqui, uma metodologia autoral desenvolvida em estudo conduzido entre os anos de 2014 e 2018, adaptada à temática da pandemia. Esta é uma versão preliminar de uma pesquisa que pretende ser ampliada, devido à novidade para o campo do planejamento urbano contemporâneo e às recorrentes mudanças da situação no Brasil.

\section{CIDADES INTELIGENTES E BIG DATA EM RESPOSTA A SITUAÇÕES CRÍTICAS}

Calvino, em sua célebre obra Le città invisibili (2002), comenta que o que de fato aproveita-se das cidades não são suas maravilhas, mas sim sua capacidade de responder a problemas. Anthopoulos (2017) reforça o argumento afirmando que uma cidade se pressupõe smart pelo atendimento efetivo das demandas locais, até mesmo quando a tecnologia não é imperativa.

Sakellarides (2020) comenta que, em tempos da COVID-19, as cidades inteligentes articulam duas mentalidades complementares. Uma é a que normatiza as decisões emergenciais nas áreas da saúde e epidemiologia, nas quais o êxito depende fundamentalmente da aderência às normas estabelecidas. Outra é a inteligência coletiva (conceito de Lévy, 2003) e colaborativa, formada pela comunidade criativa que compartilha conhecimentos para o rápido aprendizado e adaptação à situação vivida. De acordo com o autor, ser uma comunidade inteligente, em tempos como estes, significa uma adaptação veloz que aproveita o potencial digital e a disponibilidade de dados.

A mineração de dados para fins de tomada de decisão é, também, outro atributo das cidades inteligentes, e tem especial significado na situação pandêmica. Barns (2016) afirma que a gestão de dados contribui para a velocidade da resposta aos desafios por parte da governança pública. Os dados, nesse sentido, são mecanismos potencialmente capazes de promover integração entre setores, mesmo que essa integração seja "forçada" pela situação de emergência.

O uso da informação digitalizada é, para Oliver et al. (2020), uma ferramenta potencial no enfrentamento da pandemia, considerando a facilidade de disseminação de informações entre a população via telefonia móvel. Os autores apontam quatro âmbitos em que essa contribuição pode ocorrer: a) conscientização situacional, na qual a administração pública e a população podem compreender melhor as tendências baseadas em informações anteriormente ignoradas ou invisíveis; b) relação de causa e efeito das medidas tomadas, mensuração que desvela quais variáveis fazem diferença e quais causam problemas; c) previsibilidade, possível em virtude da característica de real-time do Big Data, útil na definição de ações, oportunidades, riscos e demandas; d) avaliação de impacto, que possibilita observar onde estão os obstáculos que impedem o sucesso das ações.

Inn (2020), nesta mesma linha de pensamento, afirma que o escopo de atuação das smart cities frente à pandemia revela-se, além das áreas citadas, também na utilização de inteligência artificial para monitoramento e segurança pública urbana, com uso de drones, que facilitam ações de mobilidade e de vigilância, de tecnologias de pesquisa e também de plataformas emergentes que facilitam a comunicação virtual, como chats e aplicativos. 


\section{METODOLOGIA}

A fim de traçar o panorama do ferramental digital utilizado como resposta à situação pandêmica pela administração pública nas cidades brasileiras, realizou-se um levantamento nas cem maiores cidades do país. Na seleção das cidades, foi considerado o aspecto populacional, tendo por base a projeção do Instituto Brasileiro de Geografia e Estatística para 2019 (IBGE, 2019). A opção pela consideração das grandes cidades leva em consideração o princípio de Rolnik e Solmekh (2002), que afirmam que grandes aglomerações urbanas potencializam problemáticas da gestão territorial - o que indica, para os fins deste estudo, a representatividade dos municípios selecionados. Assim, ao estudar municípios com maiores concentrações populacionais, se espera entender em escala macro - e panorâmica - de que forma as gestões municipais encaram a situação pandêmica.

A coleta de dados foi realizada entre os dias 20 e 28 de abril de 2020 com a consulta de três fontes, quais sejam (por prioridade): sites das prefeituras municipais, portais de notícias e mídias sociais das prefeituras. A busca por iniciativas com utilização do ferramental digital foi conduzida pela combinação de palavras-chave, a saber: "nome da cidade", “COVID-19", "tecnologia", "digital”, "virtual" e "smart city", aplicadas também em seus respectivos plurais e combinadas entre si com a utilização de operadores booleanos. Foram selecionados apenas os resultados oficialmente divulgados pela prefeitura municipal e que envolveram, obrigatoriamente, a tomada de decisão da gestão pública. Nesta pesquisa, considerou-se como corpus de trabalho as iniciativas que empregam o ferramental tecnológico de apoio à situação pandêmica, conforme definição de Inn (2020): monitoramento e vigilância (tais como câmeras e drones), plataformas digitais e ferramentas de comunicação (aplicativos e chats).

Os dados da busca - majoritariamente notícias - alimentaram uma planilha até a saturação de incidências. Na formatação da planilha, foram classificadas as iniciativas por finalidade de utilização da ferramenta digital. Essa classificação determinou, para cada incidência, uma palavra-chave, o que culminou, por fim, em uma categorização de vinte tipologias de análise. A atribuição das categorias deu-se pela descrição das notícias, e seguindo os recursos metodológicos de Moraes (1999) para análise de conteúdo, quais sejam: a) unitarização das informações em partes de análise; b) classificação das unidades em categorias; c) descrição e análise das categorias.

Primeiramente, a análise de dados considerou o caráter quantitativo da adoção das ferramentas digitais; em um segundo momento, o qualitativo, à luz do conceito de cidade inteligente. A fim de aprofundar a análise, considerou-se a distribuição geográfica, em comparação a dois conjuntos de dados de contexto: a) o reconhecimento municipal da adoção de ferramentas tecnológicas, indicativo de um histórico prévio de governança baseada em estratégias digitais; e b) o PIB per capita municipal. O primeiro conjunto de dados foi obtido pelo índice Connected Smart Cities (2019), que, desde 2014, elabora pesquisa anual sobre inteligenciamento urbano nos 666 municípios brasileiros com mais de 50 mil habitantes. $\mathrm{O}$ índice fornece uma classificação geral de cidades inteligentes e também as categoriza por eixos temáticos (no caso da presente pesquisa, eixo "tecnologia e inovação"), baseado na pesquisa com 70 indicadores, em convergência com outras publicações internacionais. O segundo conjunto de dados - PIB per capita - foi obtido em levantamento feito pelo IBGE, cujos resultados disponíveis mais recentes datam de 2017. 


\section{RESULTADOS}

Os resultados indicaram a adoção de ferramental digital em $83 \%$ das cidades pesquisadas. Cabe destacar, neste caso, a probabilidade de distorção (pela inexistência de casos ou pela subnotificação dos mesmos) quando não houve a divulgação da iniciativa nos meios pesquisados. Foram contabilizadas 198 diferentes estratégias digitas, incluindo o agrupamento de diversas delas em determinadas cidades.

A Figura 1 apresenta a distribuição geográfica dos resultados, indicando uma concentração nas capitais e metrópoles brasileiras, especialmente nos estados de São Paulo, Rio de Janeiro, Paraná e Distrito Federal. A Figura 2 apresenta a distribuição de casos confirmados do vírus de acordo com informações do Ministério da Saúde (2020). A comparação das duas situações sugere que há uma tendência de utilização do ferramental digital como estratégia de enfrentamento à COVID-19 justamente nas áreas mais afetadas.

Uma breve análise pelo método de Pearson mede o grau de relacionamento entre variáveis pelo intervalo -1 e +1 . Quanto mais próximo de 1, maior é a correlação positiva entre elas (Figueiredo \& Silva, 2009). Assim, medindo o número de estratégias digitais encontradas em cada estado brasileiro com os dados de casos confirmados e números de óbitos (Ministério da Saúde, 2020), temos, respectivamente, uma correlação de 0,8286 e de 0,8347 , índice considerado alto. Essa metodologia estatística é válida para índices de distribuição normal, o que, neste caso, pode apresentar certa fragilidade, já que os números variam diariamente e não necessariamente em curva constante. No entanto, em um primeiro momento, os números e a distribuição no mapa parecem sugerir uma aproximação com a hipótese "a" mencionada anteriormente: a adoção da tecnologia acompanha - ou até mesmo antecipa - a previsão de crescimento da curva de infecção. Apesar disso, é preocupante a baixa incidência de iniciativas divulgadas na região Norte e parte da região Nordeste, locais onde a ocorrência de casos confirmados é preocupante. Isso pode estar atrelado aos mais variados fatores, entre os quais os problemas de subnotificação de casos, e as questões relativas à exclusão digital - uma realidade em diversas regiões do país. Para aprofundar esta discussão, outros parâmetros devem ser acrescentados, a exemplo do que virá na sequência.

FIGURA 1 DISTRIBUIÇÃO DE RESULTADOS ENCONTRADOS

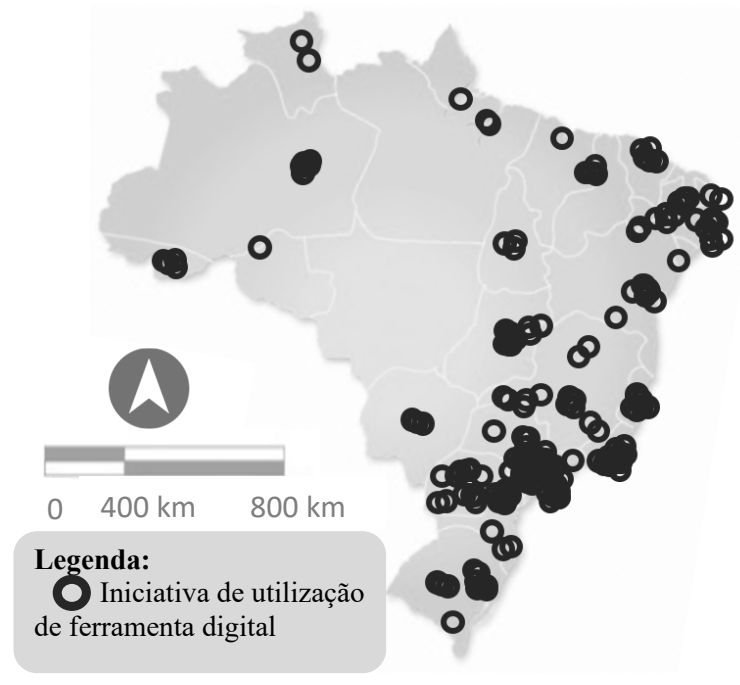

Fonte: Elaborada pela autora.

ReVISta de Administração pública | Rio de Janeiro 54(4):860-873, jul. - ago. 2020 


\section{FIGURA 2 MAPEAMENTO DE CASOS CONFIRMADOS DA COVID-19 RELATIVOS A ABRIL DE 2020}

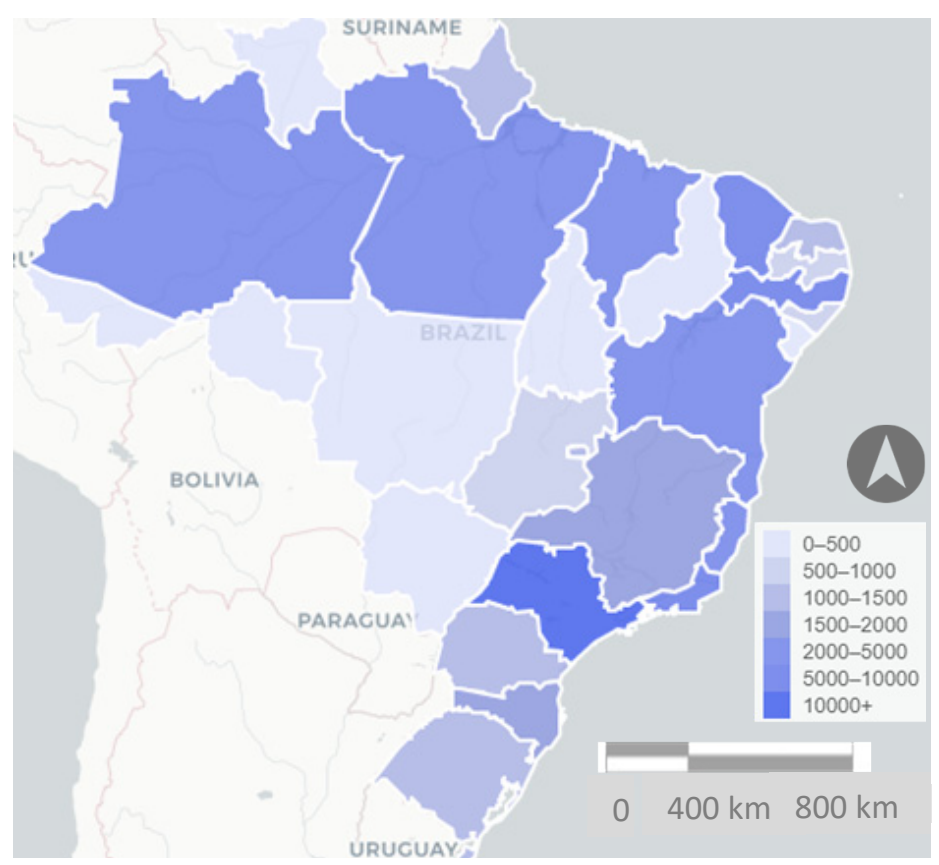

Fonte: Ministério da Saúde (2020) em 29 de abril de 2020, utilizando como base dados fornecidos pelas Secretarias de Saúde.

O processo de análise das 198 estratégias, por meio das unidades descritivas, levou à sua classificação em dez categorias, conforme apresenta o Quadro 1.

\section{QUADRO 1 DESCRIÇÃO DAS DEZ CATEGORIAS DE RESULTADOS}

\begin{tabular}{ll} 
Categoria & \multicolumn{1}{c}{ Descrição } \\
$\begin{array}{l}\text { Educação / capacitação à } \\
\text { distância }\end{array}$ & $\begin{array}{l}\text { Virtualização das aulas da rede municipal / Cursos de EaD de capacitação a servidores e } \\
\text { professores. }\end{array}$ \\
$\begin{array}{l}\text { Ferramentas digitais de } \\
\text { apoio aos hospitais /à } \\
\text { saúde }\end{array}$ & $\begin{array}{l}\text { Teleconsultas / Ferramentas para agendamento online de consultas e vacinas / Visitas virtuais } \\
\text { de pacientes infectados / Plataformas virtuais de apoio psicológico / Mapeamento de casos } \\
\text { confirmados. }\end{array}$ \\
$\begin{array}{l}\text { Reforço de informação à } \\
\text { população por via digital }\end{array}$ & $\begin{array}{l}\text { Compartilhamento de informação por meio de ferramentas digitais, tais como: envio de SMS, } \\
\text { aplicativos especificamente desenvolvidos para este fim, plataformas de monitoramento e drones. } \\
\text { Disseminação cultural via } \\
\text { Iigital }\end{array}$ \\
Incentivo municipal a artistas locais / Atividades culturais online / Divulgação cultural online em \\
substituição a eventos presenciais. \\
Central telefônica
\end{tabular}




\begin{tabular}{|c|c|}
\hline Categoria & Descrição \\
\hline $\begin{array}{l}\text { Incentivo à economia local } \\
\text { via digital }\end{array}$ & $\begin{array}{l}\text { Desenvolvimento da economia local (produtores e artesãos) por meio de disseminação virtual / } \\
\text { Transposição de feiras livres para ambiente virtual / Incentivos fiscais e auxílios à economia local } \\
\text { / Ações de fortalecimento do empreendedorismo local. }\end{array}$ \\
\hline $\begin{array}{l}\text { Monitoramento de } \\
\text { aglomeração via dados de } \\
\text { geolocalização }\end{array}$ & $\begin{array}{l}\text { Fiscalização de focos de aglomeração via dados de geolocalização obtidos por meio de dados } \\
\text { dos smartphones / Vigilância por meio de câmeras. }\end{array}$ \\
\hline Serviços municipais & Transposição de serviços e protocolos municipais para plataformas virtuais. \\
\hline $\begin{array}{l}\text { Atendimento tributário } \\
\text { online }\end{array}$ & $\begin{array}{l}\text { Transposição de atendimento tributário municipal para via virtual / simplificação de protocolos } \\
\text { tributários em meio virtual. }\end{array}$ \\
\hline Hackathon & Evento de proposição de soluções inovadoras para a situação pandêmica. \\
\hline
\end{tabular}

Fonte: Elaborado pela autora.

Observa-se que diversas cidades utilizam-se da ferramenta digital para a transposição de atividades que antes eram realizadas apenas presencialmente (como feiras locais, eventos culturais e protocolos de serviços municipais). Isso sugere que a estratégia não está exatamente servindo para otimizar uma atividade da administração pública, mas apenas à digitalização de processos. Embora essa ação vise intensificar as medidas de isolamento, ela também pode indicar uma abordagem mais elementar de aproveitamento do potencial tecnológico, o que pode ser largamente justificado pelo baixo custo da transposição do analógico para o digital, relativamente a outras melhorias. Graham e Marvin (1996) caracterizam a simples substituição do físico pelo digital como o nível mais "raso" do impacto tecnológico no meio. Este dado pode confirmar parcialmente a segunda hipótese, que indica não necessariamente uma antecipação, mas uma ação a posteriori como resposta à necessidade emergencial.

Destaca-se, também, a incidência de estratégias via central telefônica, algo aparentemente anacrônico comparado aos dispositivos digitais. No entanto, um esclarecimento rápido, via telefone, pode gerar momentaneamente um estado de confiança e conforto para o cidadão, acossado pelo estresse e pela ansiedade que caracterizam situações como a atual.

Diferentemente, o gênero de ação que se utiliza de dados provenientes de smartphones para monitoramento das aglomerações urbanas é alvo de discussões antagônicas: por um lado, a velocidade na obtenção de dados precisos por meio da geolocalização, característica das smart cities, e a utilização do Big Data para distribuição de informações e orientação da ação de administradores públicos (Lemos, 2013); por outro lado, a preocupação com a privacidade e com a proteção de identidades, aspecto que diversos pesquisadores consideram como diretriz fundamental à mensuração do projeto de inteligenciamento urbano (Aldairi \& Tawalbeh, 2017).

Dentre essas categorias, a maior incidência é do uso de ferramentas digitais como suporte educativo, a fim de manter o isolamento social sem prejuízo para os estudantes da rede municipal (ver Figura 3). Em segundo lugar, encontram-se as estratégias virtuais na área da saúde e de apoio ao sistema hospitalar, com destaque especial às teleconsultas, que evitam - pelo menos inicialmente - a aglomeração de pacientes em unidades de atendimento. Destacam-se, ainda, as iniciativas de reforço à informação por meios virtuais, especialmente por meio de aplicativos ou plataformas desenvolvidas exclusivamente para este fim. 


\section{FIGURA 3 INCIDÊNCIA DE ESTRATÉGIAS, POR CATEGORIA}

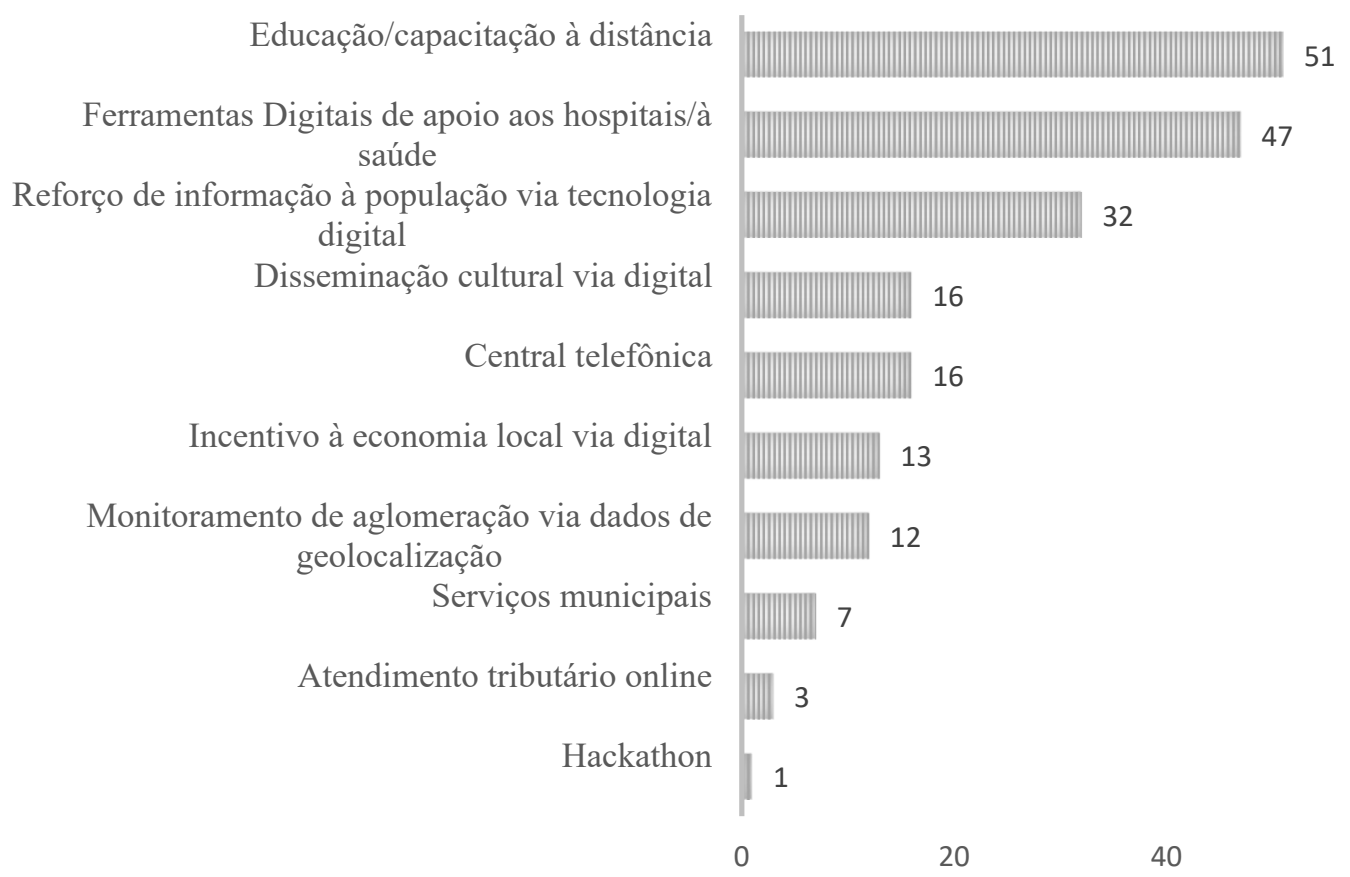


Sul e Sudeste e em parte da região Centro-Oeste, além de algumas áreas nas regiões Nordeste e Norte (especialmente capitais e regiões metropolitanas). Essa distribuição, comparativamente à disposição das iniciativas levantadas, sugere também uma tendência de adoção de ferramentas digitais em locais com certo desenvolvimento socioeconômio - aspecto que poderia ser aprofundado por meio de discussões sobre distribuição de renda, escalas produtivas regionais, escolaridade e acesso à educação.

FIGURA 4 COMPARATIVO ENTRE LEVANTAMENTO DE INICIATIVAS (A), HISTÓRICO DE ADOÇÃO DO FERRAMENTAL TECNOLÓGICO (B) E PIB PER CAPITA MUNICIPAL (C)

(a)

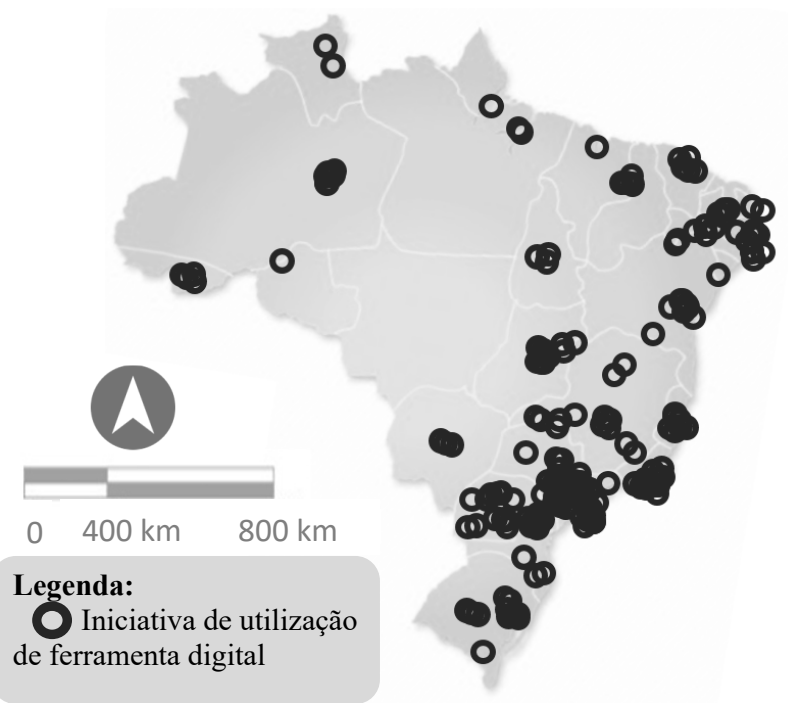

Fonte: Elaborada pela autora.

(b)

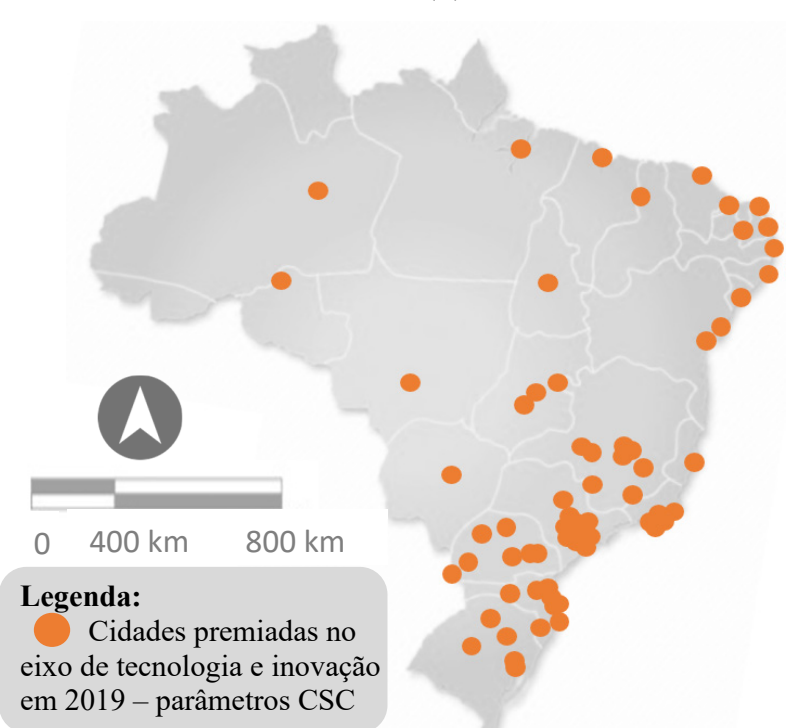

Fonte: Elaborada pela autora com base em Connected Smart Cities (2019). 
(c)

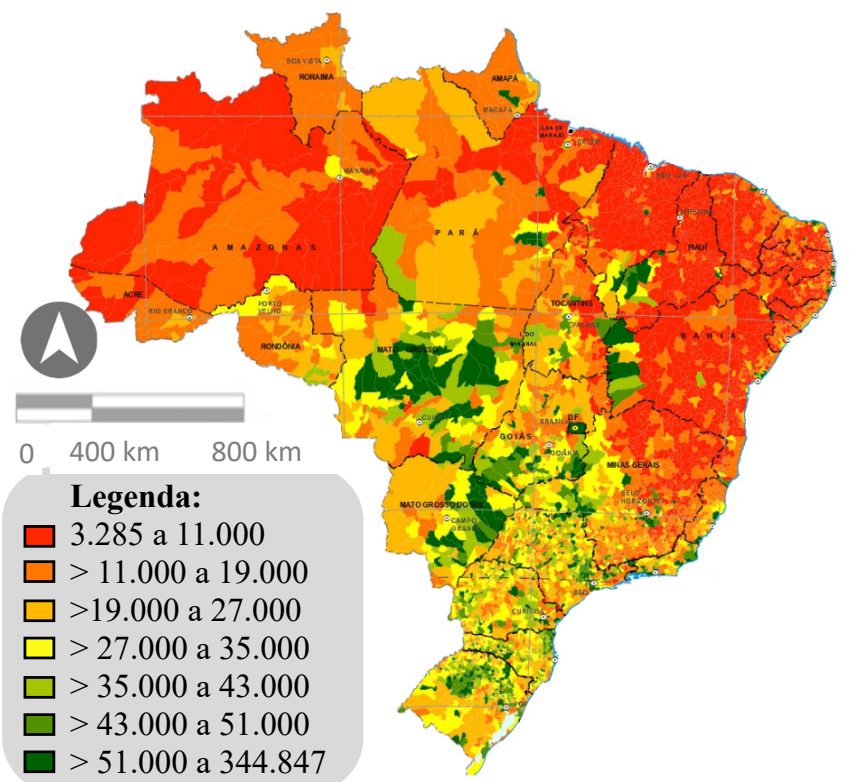

Fonte: Adaptada pela autora com base em PIB per capita em média municipal (IBGE, 2017).

Ao distribuir geograficamente as estratégias conforme categorias (Figura 5), algumas inferências podem ser feitas. Ocorre uma distribuição mais homogênea de iniciativas digitais de apoio à saúde e à educação.

O apoio à saúde (cujos resultados aparecem distribuídos no mapa d) é, nesse momento da situação pandêmica, a área de atuação mais lógica e emergencial das gestões municipais. Nesse sentido, as estratégias digitais adotadas nos municípios associam-se a outras medidas que já vêm sendo utilizadas especificamente pelos setores médicos e linhas de frente. Também aqui fica mais evidente a aproximação entre estratégias de apoio à saúde e as cidades com melhor histórico de adoção de ferramentas digitais (Figura 5), o que valida a transposição analógico-digital nos locais que já possuem alguma experiência. Ocorre o mesmo quando observamos as categorias de iniciativas no mapa (a), que denotam a adaptação de serviços e ações diversas ao modelo digital.

No caso da educação (mapa c), a alta incidência dos resultados, bem como a homogeneidade refletem a necessidade de criação de alternativas para a organização escolar municipal, mantendose, contudo, o isolamento social. Muito embora, de modo geral, a literatura não trate suficientemente do elemento educacional no processo de "inteligenciamento" urbano, pesquisadores como Komninos (2011) e Caragliu, Del Bo, e Nijkamp (2011) consideram que a cidade dita "smart" reconhece as evoluções tecnológicas e as assume também pela necessidade de disseminação do conhecimento, como no caso apresentado nos processos de capacitação e na virtualização de práticas pedagógicas. Numa perspectiva ainda mais ampla, a discussão permite uma aproximação com o conceito da inteligência coletiva de Lévy (2003), que preconiza um entendimento coletivo que é cada vez mais aprimorado conforme aprendizados retirados de experiências anteriores e validação estratégica (especialmente no sentido de antecipação às demandas, conforme a primeira hipótese postulada). 
(a)

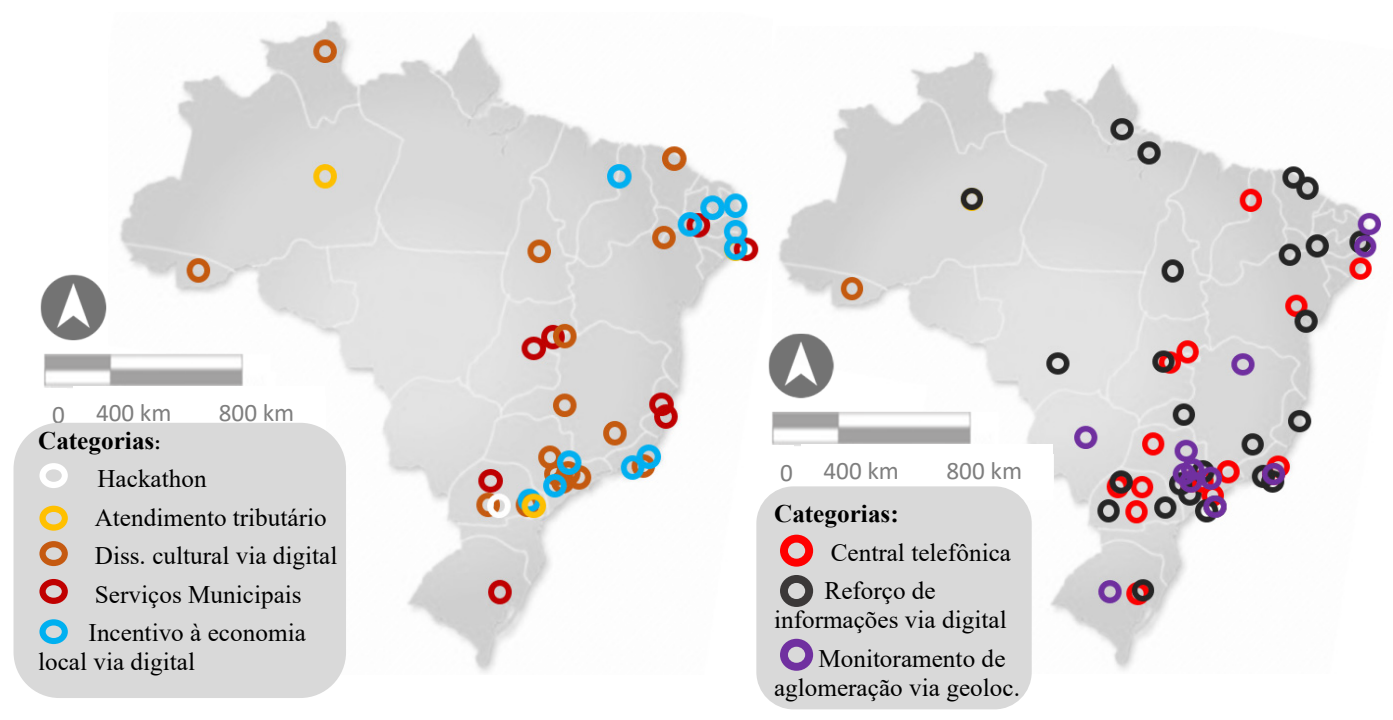

(c)

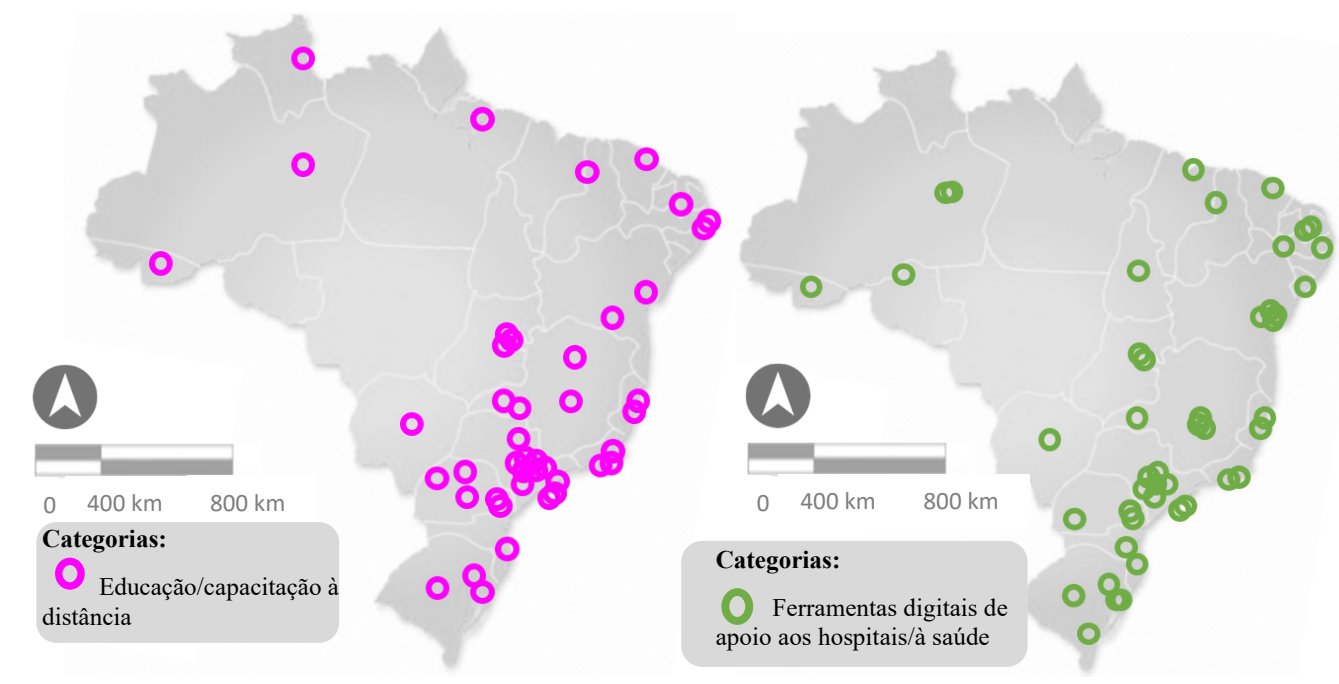

(b)

(d)

Fonte: Elaborada pela autora.

O mapa (b) apresenta as categorias relativas ao monitoramento e à disseminação de informações sobre a situação pandêmica. A distribuição geográfica das estratégias também segue a tendência dos municípios com reconhecido histórico de utilização digital. Além disso, essas categorias refletem uma das possibilidades de atuação comunicacional postulada por Oliver et al. (2020), supracitada: a conscientização situacional. Sobre os outros fatores discutidos pelos mesmos autores, alguns comentários podem ser feitos: a questão da previsibilidade não pode ser totalmente sugerida apenas pelas análises aqui apresentadas, uma vez que é preciso levar em conta a realidade de cada município 
e sua respectiva capacidade de adaptação e as tendências dos locais já testados nessa área - gerando novamente a dualidade entre o que pode ser antecipação e o que pode ser mera resposta emergencial. Quanto aos fatores de relação causa/efeito e de avaliação de impacto, a mensuração só poderá ser realizada e validada plenamente no futuro.

\section{CONSIDERAÇÕES FINAIS}

Esta pesquisa investigou as estratégias digitais identificadas nas cidades brasileiras de uma ótica de acompanhamento ou de antecipação governamental à situação pandêmica ou como uma mera resposta, obrigatória e emergencial, ao contexto. Observou-se que as tipologias identificadas seguem parcialmente os pressupostos de Oliver et al. (2020) e Inn (2020) em menção sobre as possibilidades de adoção do digital frente à crise, especialmente no que se refere à comunicação e adaptação ao ferramental virtual. Isso sugere que as cidades brasileiras pesquisadas estão seguindo, em algum nível, as tendências de otimização e de enfrentamento adotadas mundialmente.

Porém a pesquisa ressaltou que existe uma significativa tendência de as estratégias serem aplicadas exatamente nos locais em que há um contexto facilitador a esse desenvolvimento: maior PIB per capita ou histórico já reconhecido de instrumentação digital e inovadora, o que reforça a hipótese b.

No entanto, a discussão sobre o potencial tecnológico em cidades não deve ser unilateral, considerando a tecnologia como solução última para demandas. Nesse sentido, assume-se que a noção de cidade inteligente varia de acordo com o contexto. Assim sendo, a inteligência está mais atrelada à aderência da solução às demandas locais do que à adoção indiscriminada da tecnologia. A real mensuração desse processo só será possível no futuro, verificada a consolidação ou a fragmentação das estratégias aqui analisadas.

Cabe, ainda, a reflexão de que a evolução do ferramental digital, bem como o aprendizado decorrente da crise suscitam a conscientização progressiva sobre o papel da tecnologia no meio urbano e sobre a relação comunidade-governança. Isso é parte do processo de conscientização coletiva, ou da criação de uma inteligência coletiva continuamente desenvolvida a partir das lições aprendidas.

A pesquisa reconhece o caráter interino dos resultados no recorte geográfico-temporal e as variações significativas que ele pode sofrer rapidamente, especialmente na situação atual. Diante disso, estudos futuros serão necessários para validar tendências, e avaliar a perenidade de ações administrativas públicas que oportunizaram a crise como incremento de efetividade. 


\section{REFERÊNCIAS}

Abella, A., Ortiz-de-Urbina-Criado, M., DePablos-Heredero, C. (2015, novembro/dezembro). Information reuse in smart cities' ecosystems. El profesional de la información, 24(6), 838-844.

Albino, V., Berardi, U., \& Dangelico, R. M. (2015). Smart Cities: Definitions, Dimensions, Performance, and Initiatives. Journal of Urban Technology, 22(1), 3-21.

Aldairi, A. \& Tawalbeh, L. (2017). Cyber Security Attacks on Smart Cities and Associated Mobile Technologies. Procedia Computer Science, 109, 1086-1091. Recuperado de https://doi.org/10.1016/j. procs.2017.05.391

Amin, A. \& Thrift, N. (2002). Cities: reimagining the urban. New Jersey, NJ: Wiley.

Angelidou, M. (2014, July). Smart city policies: a spatial approach. Cities, 41(S1), S3-S11. Recuperado de https://doi.org/10.1016/j.cities.2014.06.007

Anthopoulos, L. (2017). Smart utopia VS smart reality: learning by experience from 10 smart city cases. Cities, 63, 128-148. Recuperado de https://doi. org/10.1016/j.cities.2016.10.005

Barns, S. (2016). Mine your data: open data, digital strategies and entrepreneurial governance by code. Urban Geography, 37, 557-571.

Bibri, S. E., \& Krogstie, J. (2017). Smart sustainable cities of the future: an extensive interdisciplinary literature review. Sustainable Cities and Society, 31, $183-212$.

Calvino, I. (2002). Le città invisibili. Milano, Italia: Oscar Mondadori.

Caragliu, A., Del Bo, C., \& Nijkamp, P. (2011). Smart Cities in Europe. Journal of Urban Technology, 18(2), 65-82.

Cassandras, C. G. (2016). Smart Cities as CyberPhysical Social Systems. Engineering, 2, 156-158.

Connected Smart Cities (2019). Ranking Connected Smart Cities 2019. Recuperado de https://www. connectedsmartcities.com.br/ranking-o-que-e/

Graham, S., \& Marvin, S. (1996). Telecommunications and the city: electronic spaces, urban places. London, UK: Routledge.
Inn, T. L. (2020, 27 de março). Smart City Technologies Take on COVID-19 (Penang Institute Issues: analysing Penang, Malasya and the region). Penang, Malásia: Penang Institute. Recuperado de https://penanginstitute.org/wp-content/ uploads/2020/04/27_03_2020_LII-INN.pdf

Instituto Brasileiro de Geografia e Estatística. (2017). PIB dos municípios: PIB per capita 2017. Rio de Janeiro, RJ: Autor. Recuperado de https://www.ibge. gov.br/apps/pibmunic/

Instituto Brasileiro de Geografia e Estatística. (2019). Estimativas de população 2019. Rio de Janeiro, RJ: Autor. Recuperado de https://www.ibge.gov.br/ estatisticas/sociais/populacao/9103-estimativas-depopulacao.html? $=\& \mathrm{t}=\mathrm{o}$-que-

Figueiredo, D. B., Filho, \& Silva, J. A., Jr. (2009). Desvendando os Mistérios do Coeficiente de Correlação de Pearson (r). Revista Política Hoje, 18(1), 115-146.

Komninos, N. (2011). Intelligent cities: variable geometries of spatial intelligence. Intelligent Buildings International, 3, 172-188.

Lemos, A. (2013). Cidades inteligentes: de que forma as novas tecnologias - como a computação em nuvem, o Big Data e a Internet das Coisas - podem melhorar a condição de vida nos espaços urbanos? Gvexecutivo, 12(2), 46-49.

Lévy, P. (2003). A inteligência coletiva: por uma antropologia do ciberespaço. (4. ed.). São Paulo, SP: Loyola.

Llacuna, M. M., Colomer-llinàs, J., \& MeléndezFrigola, J. (2015). Lessons in urban monitoring taken from sustainable and livable cities to better address the Smart Cities initiative. Technological Forecasting \& Social Change, 90, 611-622.

Ministério da Saúde. (2020). Coronavírus Brasil. Brasília, DF: Autor. Recuperado de https://covid. saude.gov.br/

Moraes, R. (1999). Análise de conteúdo. Revista Educação, 22(37), 7-32.

Oliver, N., Letouzé, E., Sterly, H., Delataille, S., De Nadai, M., Lepri, B. ... Vinck, P. (2020). Mobile phone data and COVID-19: Missing an opportunity? Ithaca, NY: Cornell University. Recuperado de https://arxiv. org/abs/2003.12347 
RAP | Smart cities e pandemia: tecnologias digitais na gestão pública de cidades brasileiras

Organização Mundial da Saúde. (2020). Nuevo Coronavirus 2019. Recuperado de https:// www.who.int/es/emergencies/diseases/novelcoronavirus-2019

Rolnik, R., \& Somekh, N. (2002). Governar as metrópoles: dilemas da recentralização. São Paulo em Perspectiva, 14(4), 83-90.

Sakellarides, C. (2020). From Viral City to Smart City: Learning from Pandemic Experiences. Acta
Médica Portuguesa, 33(6), 359-361. Recuperado de https://www.actamedicaportuguesa.com/revista/ index.php/amp/article/view/13841/5927

Salgado, M. M. (2016). A inteligência na sociedade positiva: dos humanos às cidades. In L. Santaella. (Org.), Cidades inteligentes: por que, para quem? (pp. 38-49). São Paulo, SP: Estação das Letras e Cores.

TrandingView. Coronavírus (COVID-19) gráficos e estatísticas. Recuperado de https://br.tradingview. com/covid19/

\section{Tharsila Maynardes Dallabona Fariniuk}

https://orcid.org/0000-0002-2283-0692

Doutora em Gestão Urbana pelo Programa de Pós Graduação em Gestão Urbana da Pontifícia Universidade Católica do Paraná (PUCPR); Pesquisadora em nível de pós-doutorado em Gestão Urbana pela Pontifícia Universidade Católica do Paraná (PUCPR); Professora no Centro Universitário Unifacear do Curso de Arquitetura e Urbanismo. E-mail: tharsilamd@gmail.com 